\title{
Zu langsame Therapieeskalation erhöht Infarkt- und Insult-Risiken drastisch
}

\author{
Was ist schlimmer für den Diabeti- \\ ker: Der plötzliche Herztod in 20 Jahren \\ oder ein kontinuierliches mikrovaskulä- \\ res Desaster mit Impotenz, Abnahme \\ der Sehkraft, Gangunsicherheit, \\ reduzierter Belastbarkeit und chroni- \\ schen neuropathischen Schmerzen? \\ Mit dieser rhetorischen Frage wollte \\ Prof. Dirk Müller-Wieland, Hamburg, \\ ins Gedächtnis rufen, dass mikrovasku- \\ läre Diabeteskomplikationen die Le- \\ bensqualität oft über lange Zeit stark \\ beeinträchtigen können. Auch korrelie- \\ re die Qualität der Blutzuckereinstellung \\ schon mittelfristig mit diesen Kompli- \\ kationen. Ein Vorteil hinsichtlich ma- \\ krovaskulärer Komplikationen stelle \\ sich hingegen oft erst langfristig ein.
}

Müller-Wieland empfahl eine proaktive und keine reaktive Intensivierung der antidiabetischen Therapie. Wenn immer erst dann reagiert werde, wenn die Blutzuckerwerte bereits eine Weile deutlich $\mathrm{zu}$ hoch liegen, riskiere der $\mathrm{Pa}$ tient frühzeitig beides - mikro- und makrovaskuläre Katastrophen.

Als Beleg zitierte er eine retrospektive Studie mit 105.477 neu diagnostizierten Typ-2-Diabetikern [Paul SK et al. Cardiovasc Diabetol. 2015;14:100]. Verglichen wurden Patienten, deren $\mathrm{HbA}_{1 \mathrm{c}}{ }^{-}$ Wert in den ersten zwei Jahren konstant über 7,5\% lag, mit frühzeitig gut eingestellten Patienten $\left(\mathrm{HbA}_{1 \mathrm{c}}\right.$-Wert $\left.<7 \%\right)$. Die Autoren errechneten Risikoerhöhungen von $80 \%$ für Herzinfarkte, 50\% für Schlaganfälle und 63\% für Herzinsuffizienz für jedes Jahr, in dem die notwendige Therapieintensivierung verschlafen wurde.

Vor allem bei relativ jungen Typ2-Diabetikern sei eine straffe und sichere $\mathrm{HbA}_{1 \mathrm{c}}$-Kontrolle wichtig. „Ich empfehle Lebensstilmaßnahmen plus Metformin und als nächste Eskalationsstufen DPP-4-Hemmer (z. B. Januvia ${ }^{\circledR}$ ) und/ oder SGLT-2-Hemmer“, so Müller-Wieland. Mit diesen Medikamenten nimmt der Patient nicht zu und minimiert sein Hypoglykämierisiko.

\section{Dr. Dirk Einecke}

- Symposium „Mikro- und makrovaskuläre Folgeschäden vermeiden - evidenzbasierte Therapie für I hre Patienten mit Typ2-Diabetes", 51. Kongress der Deutschen Diabetes Gesellschaft; Berlin, Mai 2016 (Veranstalter:MSD)

\section{Grippeimpfung}

\section{Wirkverstärkter Impfstoff für Senioren}

— „Influenza ist eine Systemerkrankung“, sagte Prof. Barbara Gärtner, Homburg. Influenza erhöhe das Risiko für Schlaganfall, Pneumonie, Herzinfarkt sowie Myokarditis und verschlimmere Erkrankungen wie COPD und Asthma und die chronische Herzkrankheit. Gerade bei älteren Menschen, die häufig unter einer oder mehrerer dieser Vorerkrankungen leiden, komme es dann zu einem schlimmeren Verlauf, zur Hospitalisation und zu erhöhter Sterblichkeit.

Je älter die Menschen, desto niedriger sind aber die Antikörpertiter nach einer Impfung, erläuterte Gärtner. Das Schlüsselwort dazu heiße Immunseneszenz. Um den altersbedingten Veränderungen des Immunsystems Rechnung zu tragen, wurde bereits vor etwa 20 Jahren mit Fluad ${ }^{\circledast}$ ein spezieller Impfstoff entwickelt. Der Impfstoff enthält einen Wirkverstärker, der die Immunantwort verbessern kann. „Dadurch kommen deutlich mehr Makrophagen an den Ort der Impfung“, so Dr. Petra Sandow, Berlin. Mehr Makrophagen bedeuten mehr Antigen-präsentierende Zellen, und im Endeffekt mehr Antikörper. Diese Antikörper setzen sich dann an das Hämagglutinin der Viren.

Belegt wurde die Wirksamkeit des Impfstoffs unter anderem in der LIVEStudie mit über 170.000 mindestens 65-Jährigen in Norditalien in der jeweiligen Grippesaison der Jahre 2006-2009. Bei mit Fluad ${ }^{\circledR}$ Geimpften lag die Rate an Influenza-bedingten Krankenhauseinweisungen im Vergleich mit einem herkömmlichen trivalenten Impfstoff um 25\% niedriger. Die Studienergebnisse wurden auch vom Paul-Ehr

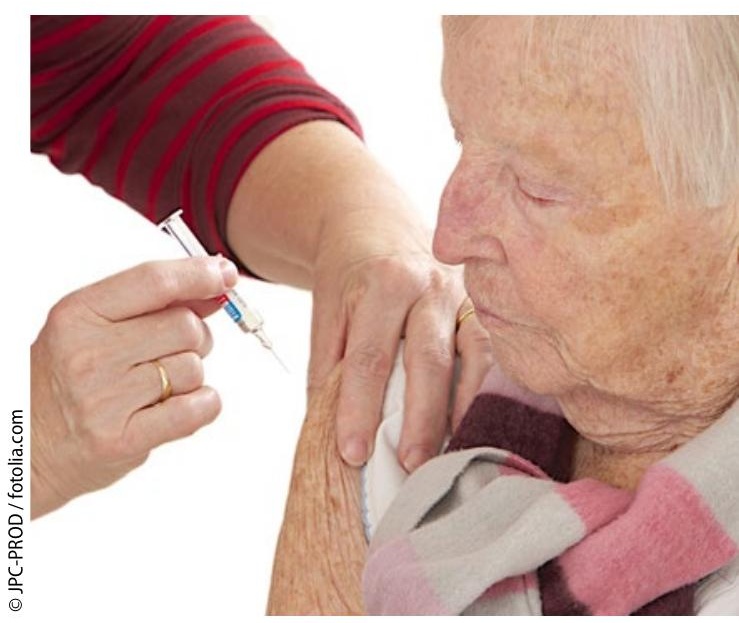

Gegen Grippe geimpft werden - das ist vor allem für Ältere wichtig.

lich-Insitut anerkannt und sind seit diesem Jahr in die Fachinformation von Fluad $^{\oplus}$ aufgenommen.

\section{Christine Ott}

- Pressekonferenz "Fluad ${ }^{\circledast}$ - ein besonderer Impfstoff für einen besonderen Lebensabschnitt"; Frankfurt a. M., August 2016 (Veranstalter Sequirus) 